Supporting information for

\title{
Intrinsically Stretchable n-Type Polymer Semiconductors through Side Chain Engineering
}

Yafei Ding,,$\dagger$ Ye Yuan,,+ Ning Wu, $\dagger$ Xiaohong Wang,,$\dagger$ Guobing Zhang, ${ }^{*}, \dagger$ Longzhen $\mathrm{Qiu}^{*}, \uparrow^{\perp}$

$\dagger$ National Engineering Lab of Special Display Technology, State Key Lab of Advanced Display Technology, Academy of Opto-Electronic Technology, Hefei University of Technology, Hefei 230009, China

$\$$ Anhui Province Key Laboratory of Advanced Functional Materials and Devices, Hefei University of Technology, Hefei 230009, China

${ }^{\perp}$ Anhui Province Key Laboratory of Measuring Theory and Precision Instrument, Hefei University of Technology, Hefei 230009, China

E-mail: gbzhang@hfut.edu.cn, lzhqiu@hfut.edu.cn

Keywords: polymer semiconductors; side chain engineering; aggregate-state structures; electron mobility, mechanical properties 


\section{Table of Contents}

- General methods.

- Synthesis of monomers and polymers.

- Fabrication and characterization of polymer-based OFETs

- Solubility tests.

- GPC of CC-PBIBDF, Si-PBIBDF, and CSi-PBIBDF.

- TGA of CC-PBIBDF, Si-PBIBDF, and CSi-PBIBDF.

- DSC of CC-PBIBDF, Si-PBIBDF, and CSi-PBIBDF.

- Temperature-dependent UV-vis-NIR of CC-PBIBDF, Si-PBIBDF, and CSi-PBIBDF.

- CV of CC-PBIBDF, Si-PBIBDF, and CSi-PBIBDF.

- AFM height images of as-cast polymer films.

- GIWAXS-2D patterns of as-cast polymer films.

- GIWAXS-1D curves of as-cast and annealed polymer films.

- Transfer characteristics curves of the OFETs based-on the polymer films.

- Output characteristics curves of the OFETs based-on the polymer films.

- Fabrication process of OFETs with the stretched polymer fims.

- OM images of the buckled polymer films.

- Transfer characteristics curves of the printed polymer films of CSi-PBIBDF after multiple stretching-releasing cycles at $50 \%$ strain. 


\section{General methods}

Nuclei magnetic resonance (NMR) spectra were recorded on an Agilent VNMRS600 spectrometer $(600 \mathrm{MHz})$. Elemental analysis was measured on a Vario EL instrument. Number average molecular weight $(M \mathrm{n})$, weight average molecular weight $(M \mathrm{w})$, and polydispersity index (PDI) were determined by gel permeation chromatography (GPC) equipped with a Waters 1515 pump and a Waters 2414 differential refractive index detector. The eluent used was chloroform at a temperature of $35^{\circ} \mathrm{C}$ and a flow rate of $1 \mathrm{~mL} \mathrm{~min}{ }^{-1}$. Thermogravimetric analysis (TGA) was carried out on a TA Instrument QS000IR. Differential scanning calorimetry (DSC) were performed on a Instrument DSC Q200 calorimeter under a nitrogen atmosphere and a heating/cooling rate of $30{ }^{\circ} \mathrm{C}$ $\mathrm{min}^{-1}$. Cyclic voltammetry (CV) measurements were conducted on a CHI $660 \mathrm{D}$ electrochemical workstation under nitrogen equipped with a three-electrode system containing $\mathrm{Bu}_{4} \mathrm{NPF}_{6}(0.1 \mathrm{M})$ in deoxygenated anhydrous acetonitrile and at a scan rate of $100 \mathrm{mV} \mathrm{s}^{-1}$. Polymer film was coated on surface of Pt disc that acted as the working electrode, another platinum wire was used as the auxiliary electrode, meanwhile, an $\mathrm{Ag} / \mathrm{Ag}+$ electrode acted as the reference electrode, and ferrocene was selected as the standard. Ultraviolet-Visible-Near infrared (UV-Vis-NIR) spectra was performed on an Agilent Cary 5000 UV-Vis-NIR spectrophotometer with samples in both solution and thin film. The surface structure of polymer film was obtained with atomic force micrograph (AFM) using a SPA300HV instrument. Grazing-incidence wide-angle Xray scattering (GIWAXS) was performed with a wavelength of 1.54 angstroms. To avoid air scattering, data was collected at a sample-to-detector distance of $300 \mathrm{~mm}$ under vacuum. The electrical characteristics of the transistors were conducted by using a Keithley 4200-SCS semiconductor parametric analyzer under ambient atmosphere at room temperature. Thin film tensile tests were performed on a water surface through the pseudo-free-standing tensile tester, as described in the previous reports.

\section{Synthesis of monomers and polymers}

All reagents and starting materials for the reactions were purchased from Sinopharm Chemical Reagent Company, Sigma-Aldrich Chemical Company, Tokyo Chemical Industry Company, Alfa AesarCompany, and used directly without further purification. 
The monomers pentadecamethylheptasiloxane, ${ }^{[\mathrm{S} 1, \mathrm{~S} 2]}$ benzo[1,2-b:4,5-b']difuran2,6(3H,7H)-dione, ${ }^{[\mathrm{S} 3]}$ (3E,7E)-3,7-bis(6-bromo-1-(2-decyltetradecyl)-2-oxoindolin-3ylidene)benzo[1,2-b:4,5-b']difuran-2,6(3H,7H)-dione $\quad$ (BIBDF-C), $\left.{ }_{[} \mathrm{S} 4\right] \quad$ were synthesized following the reported method.

\section{Synthesis of IDT-C}

Under ice bath, sodium hydrogen $(0.6 \mathrm{~g}, 14.9 \mathrm{mmol})$ was added to a solution of 6bromoisatin $(2.6 \mathrm{~g}, 11.5 \mathrm{mmol})$ dissolved in anhydrous DMF $(20 \mathrm{~mL})$. After 20 minutes, 1-bromo hexene $(2.3 \mathrm{~g}, 14.1 \mathrm{mmol})$ was injected through a septum under nitrogen. The mixture was stirred at room temperature overnight and then poured into water $(50 \mathrm{~mL})$. The organic phase was extracted by $\mathrm{CH}_{2} \mathrm{Cl}_{2}$, washed with brine, dried over $\mathrm{MgSO}_{4}$. The organic layers were concentrated and the residue was subjected to silica gel chromatography using ethyl acetate/hexane (1:20 - 1:50) as an eluent, yielding orange solid (2.4 g, yield: 68.0\%). ${ }^{1} \mathrm{H}-\mathrm{NMR}\left(\mathrm{CDCl}_{3}, 600 \mathrm{MHz}, \mathrm{ppm}\right): \delta=7.44(\mathrm{~d}, 1 \mathrm{H}), 7.25$ (d, 1H), $7.05(\mathrm{~d}, 1 \mathrm{H}), 5.79-5.72(\mathrm{~m}, 1 \mathrm{H}), 5.03-4.96(\mathrm{~m}, 2 \mathrm{H}), 3.70-3.67(\mathrm{t}, J=8.0 \mathrm{~Hz}$, 2H), 2.13-2.09 (m, 2H), 1.71-1.66 (m, 2H), 1.50-1.45 (m, $2 \mathrm{H}) .{ }^{13} \mathrm{C}-\mathrm{NMR}\left(\mathrm{CDCl}_{3}, 150\right.$ MHz, ppm): $\delta=182.25,157.94,151.75,137.81,133.52,126.81,126.37,116.25$, $115.32,113.72,40.23,33.06,26.45,25.91$.

\section{Synthesis of IDT-Si}

IDT-C (0.9 g, $2.9 \mathrm{mmol})$ was dissolved in anhydrous toluene $(15 \mathrm{~mL})$ under a nitrogen atmosphere. Pentadecamethylheptasiloxane $(2.1 \mathrm{~g}, 3.8 \mathrm{mmol})$ was injected through a septum, followed by the addition of a drop $(50 \mu \mathrm{L})$ of karstedt's catalyst (platinumdivinyltetramethyl-siloxane complex in xylene, $3 \mathrm{wt} \%$ ). The resulting mixture was stirred at $50{ }^{\circ} \mathrm{C}$ under nitrogen till complete consumption of IDT-C, which was monitored by TLC. The solution was directly subjected to silica gel chromatography using ethyl acetate/hexane (1:50 - 1:100) as an eluent, yielding an orange oil (2.1 g, yield: $72.9 \%) .{ }^{1} \mathrm{H}-\mathrm{NMR}\left(\mathrm{CDCl}_{3}, 600 \mathrm{MHz}, \mathrm{ppm}\right): \delta=7.44(\mathrm{~d}, 1 \mathrm{H}), 7.25(\mathrm{~d}, 1 \mathrm{H}), 7.04$ (d, $1 \mathrm{H}), 3.68-3.66(\mathrm{t}, J=8.1 \mathrm{~Hz}, 2 \mathrm{H}), 1.68-1.65(\mathrm{~m}, 2 \mathrm{H}), 1.42-1.35(\mathrm{~m}, 6 \mathrm{H}), 0.54-0.51$ (m, 2H), 0.07-0.03 (m, 45H). ${ }^{13} \mathrm{C}-\mathrm{NMR}\left(\mathrm{CDCl}_{3}, 150 \mathrm{MHz}, \mathrm{ppm}\right): \delta=182.30,157.91$, $151.83,133.47,126.75,126.33,116.25,113.70,40.47,32.89,27.13,26.54,23.07$, 18.14 . 


\section{Synthesis of BIBDF-Si}

To a solution of IDT- Si $(2.0 \mathrm{~g}, 2.4 \mathrm{mmol})$ in anhydrous toluene $(20 \mathrm{~mL})$, benzo[1,2b:4,5-b']difuran-2,6(3H,7H)-dione $(228 \mathrm{mg}, 1.2 \mathrm{mmol})$ and toluenesulfonic acid monohydrate ( $23 \mathrm{mg}, 0.12 \mathrm{mmol}$ ) was added. After reflux under argon atmosphere for $12 \mathrm{~h}$, the mixture was cooled to room temperature and and then poured into water (50 $\mathrm{mL}$ ). The organic phase was extracted by $\mathrm{CH}_{2} \mathrm{Cl}_{2}$, washed with brine, dried over $\mathrm{MgSO}_{4}$. After removal of the solvents under reduced pressure, the residue was purified by silica gel chromatography using ethyl acetate/hexane $(1: 20-1: 50)$ as an eluent, yielding a dark solid $(0.2 \mathrm{~g}$, yield: $11.2 \%) .{ }^{1} \mathrm{H}-\mathrm{NMR}\left(\mathrm{CDCl}_{3}, 600 \mathrm{MHz}, \mathrm{ppm}\right): \delta=8.94$ (s, 2H), $8.80(\mathrm{~d}, 2 \mathrm{H}), 7.08$ (d, 2H), $6.80(\mathrm{~s}, 2 \mathrm{H}), 3.69-3.67(\mathrm{t}, 4 \mathrm{H}), 1.68-1.65(\mathrm{~m}, 4 \mathrm{H})$, 1.42-1.35 (m, 12H), 0.54-0.51 (m, 4H), 0.07-0.03 (m, 90H). ${ }^{13} \mathrm{C}-\mathrm{NMR}\left(\mathrm{CDCl}_{3}, 150\right.$ MHz, ppm): $\delta=166.96,166.78,151.68,146.75,135.55,131.54,128.96,126.72$, 126.36, 125.55, 119.68, 111.66, 110.97, 40.34, 32.81, 27.09, 26.72, 22.97, 18.18, 1.76, $1.18,1.12,1.04,0.16$.

\section{Stille Polymerization}

To an air-free flask, monomer BIBDF-C or BIBDF-Si $(0.066 \mathrm{mmol})$ and 5,5'bis(trimethylstannyl)-2,2'-bithiophene or 5,5'-bis(tributylstannyl)-3,3'-bis(dodecyl)2,2'-bithiophene (0.066 mmol) were added under nitrogen followed by tris(dibenzylidenacetone)dipalladium (0.00264 mmol) and tri(o-tolyl) phosphine (0.01056 mmol). The flask and its contents were subjected to 3 freeze pumping/ nitrogen blowing cycles followed by addition of anhydrous chlorobenzene $(10 \mathrm{~mL})$ via syringe. The sealed reaction mixture was stirred at $130{ }^{\circ} \mathrm{C}$ for 48 hours. After cooled to room temperature, the mixture was dripped into $100 \mathrm{~mL}$ stirred methanol (containing 5 $\mathrm{mL} 12 \mathrm{M}$ hydrochloride acid). After stirring for 2 hours, the precipitated solid was collected by centrifugation and decantation. After filtration and drying under reduced pressure, the polymers were subjected to sequential Soxhlet extraction. The product was purified by washing with methanol, acetone, hexane, dichloromethane, and chloroform in a Soxhlet extractor. C-PBIBDF was almost insoluble in these organic solvents. CC-PBIBDF, Si-PBIBDF, and CSi-PBIBDF were mostly extracted with hot chloroform in an extractor for $12 \mathrm{~h}$. 
(CC-PBIBDF). Isolated yield $=81.6 \% . \mathrm{Mn}=147.0 \mathrm{kDa}, \mathrm{Mw}=377.9 \mathrm{kDa}, \mathrm{PDI}=2.57$. For C106H158N2O6S2: C, 78.61; H, 9.76; N, 1.73; S,3.95. Found: C, 77.50; H, 9.61; N, 1.93; S,4.09

(Si-PBIBDF). Isolated yield $=78.1 \% . \mathrm{Mn}=201.3 \mathrm{kDa}, \mathrm{Mw}=471.0 \mathrm{kDa}, \mathrm{PDI}=2.34$. For C76H126N2O18S2Si14: C, 50.38; H, 6.96; N, 1.54; S,3.53. Found: C, 48.76; H, 6.72; N, 1.76; S,3.72.

$($ CSi-PBIBDF $)$. Isolated yield $=75.2 \% . \mathrm{Mn}=109.7 \mathrm{kDa}, \mathrm{Mw}=314.9 \mathrm{kDa}, \mathrm{PDI}=2.87$. For C100H174N2O18S2Si14: C, 55.91; H, 8.11; N, 1.30; S,2.98. Found: C, 55.75; H, $8.21 ; \mathrm{N}, 1.51 ; \mathrm{S}, 3.08$

\section{Fabrication and characte rization of polymer-based OFET}

BGTC OFETs were fabricated to characterize the charge transport properties of all PBIBDF polymers. An n-doped $\mathrm{Si}$ and $300 \mathrm{~nm}$-thick $\mathrm{SiO}_{2}$ was used as the gate electrode and dielectric layer, respectively. The polymer solution $\left(5 \mathrm{mg} \mathrm{mL}^{-1}\right)$ was added dropwise onto the fluoropolymer (CYTOP)-treated $\mathrm{Si} / \mathrm{SiO}_{2}$ and spin-coated at $4000 \mathrm{rpm}$ for $30 \mathrm{~s}$. The polymer film was subsequently annealed at different temperatures $\left(90,120,150,180,210,240{ }^{\circ} \mathrm{C}\right)$ for $30 \mathrm{~min}$ under nitrogen. The source and drain electrodes (40 nm-thick $\mathrm{Au})$ were prepared by thermal evaporation, and the channel length (L) and width (W) were 100 and $1000 \mu \mathrm{m}$, respectively. The electrical characteristics of the OFETs were measured under vacuum conditions by using a Keithley 4200-SCS semiconductor parametric analyzer. The electron mobilities were calculated according to the conventional equation in the saturation regime at the saturation regime.

$$
I_{d}(\mathrm{sat})=\frac{W}{2 L} \cdot C_{i} \cdot \mu_{\mathrm{sat}} \cdot\left(V_{g}-V_{\mathrm{th}}\right)^{2}
$$

where $\mathrm{I} d$ is the drain current in saturated regime, W/L is the channel width/length, $\mathrm{C}_{i}$ is the gate dielectric capacitance per unit area, $\mathrm{V}_{g}$ and $\mathrm{V}_{\text {th }}$ are the gate voltage and the threshold voltage, respectively. 


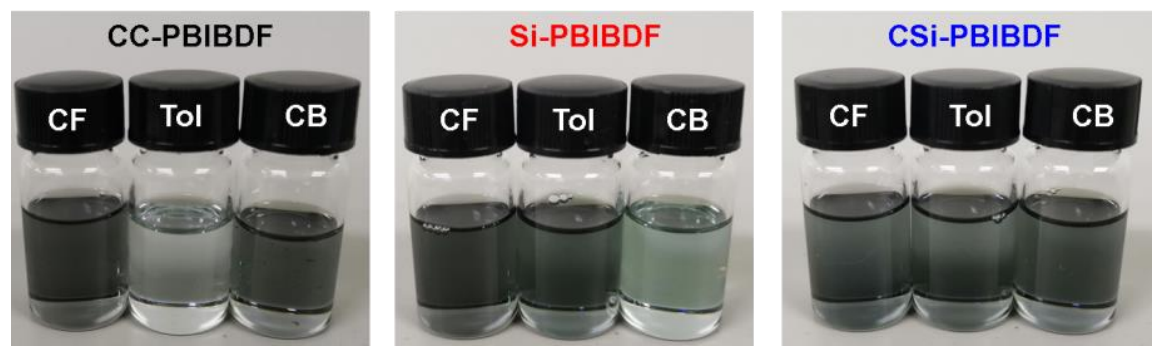

Figure S1. Solubility test of PBIBDF polymers in chloroform (CF), toluene (Tol), and chlorobenzene $(\mathrm{CB})$ at room temperature. Polymer solutions $\left(\sim 10^{-5} \mathrm{~g} / \mathrm{mL}\right)$ were filtere using filter with pore size of $200 \mathrm{~nm}$.
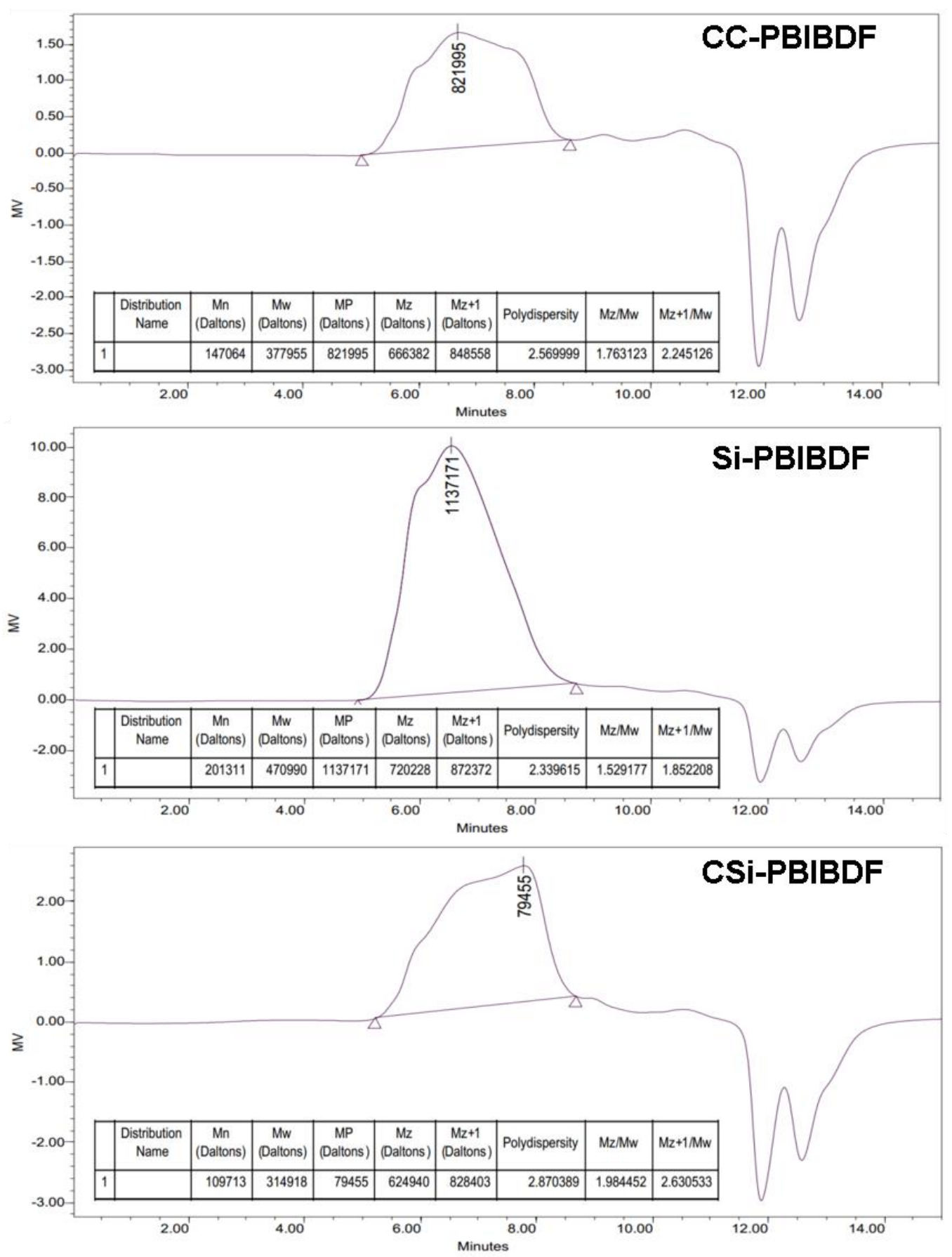
Figure S2. GPC results at $30^{\circ} \mathrm{C}$ with chloroform as eluent for CC-PBIBDF, Si-PBIBDF, and CSi-PBIBDF.

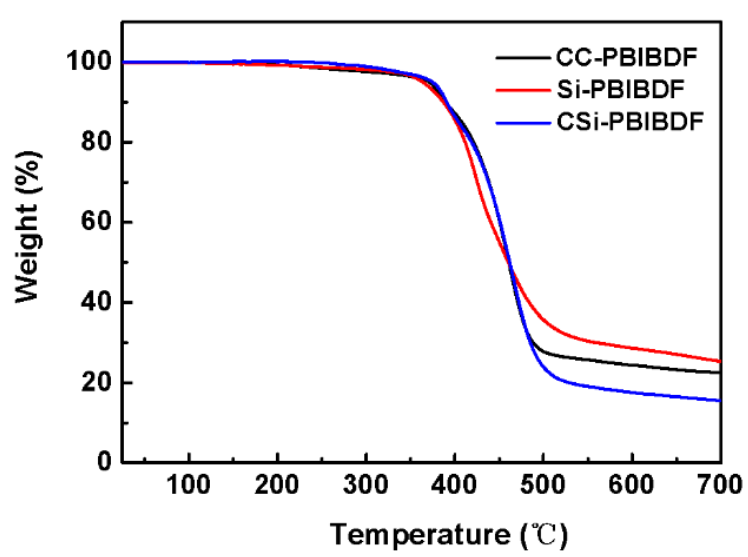

Figure S3. TGA analysis of CC-PBIBDF, Si-PBIBDF, and CSi-PBIBDF.

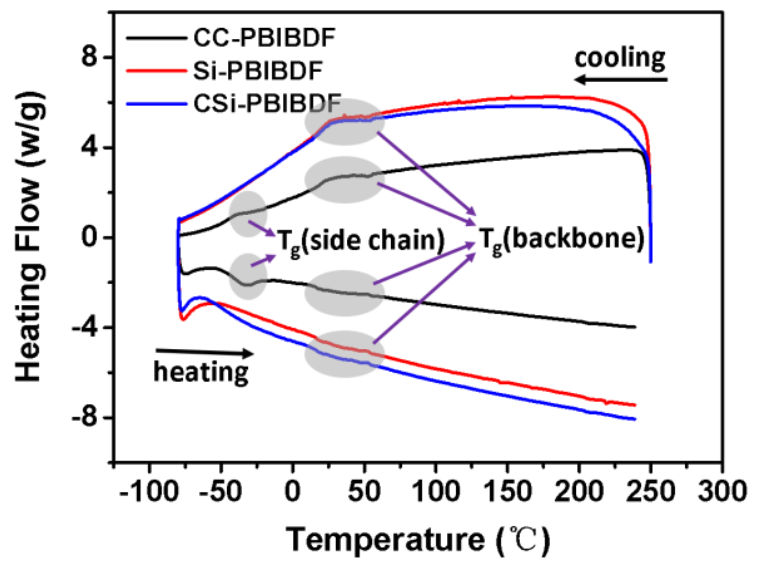

Figure S4. DSC analysis of CC-PBIBDF, Si-PBIBDF, and CSi-PBIBDF.
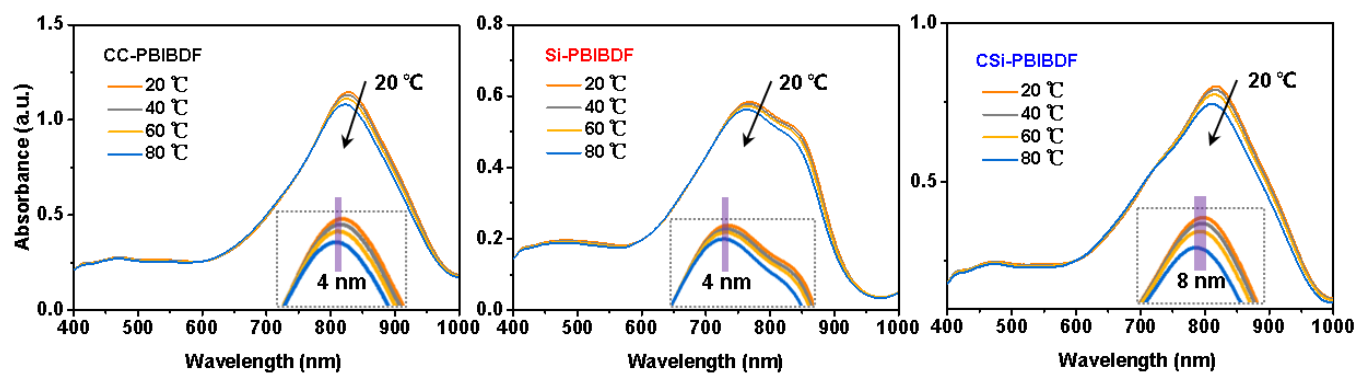

Figure S5. Temperature-dependent UV-vis-NIR absorption spectra of CC-PBIBDF, Si-PBIBDF, and CSi-PBIBDF in diluted chlorobenzene solutions. 

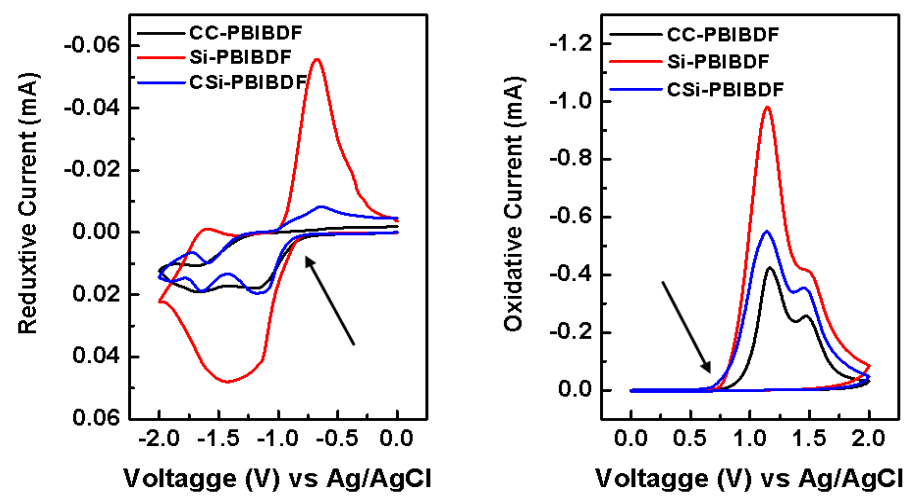

Figure S6. CV measurement of CC-PBIBDF, Si-PBIBDF, and CSi-PBIBDF.
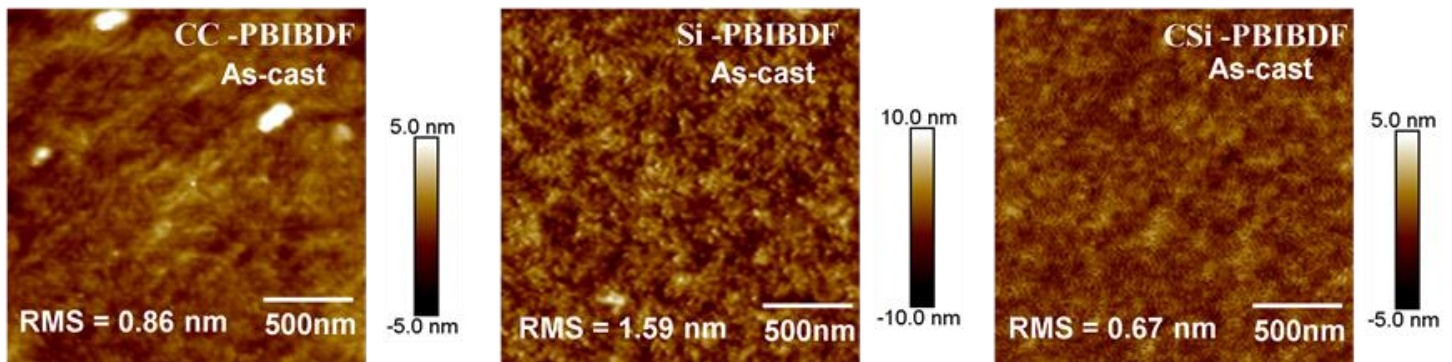

Figure S7. AFM height images for as-cast polymer thin-films of CC-PBIBDF, SiPBIBDF, and CSi-PBIBDF.
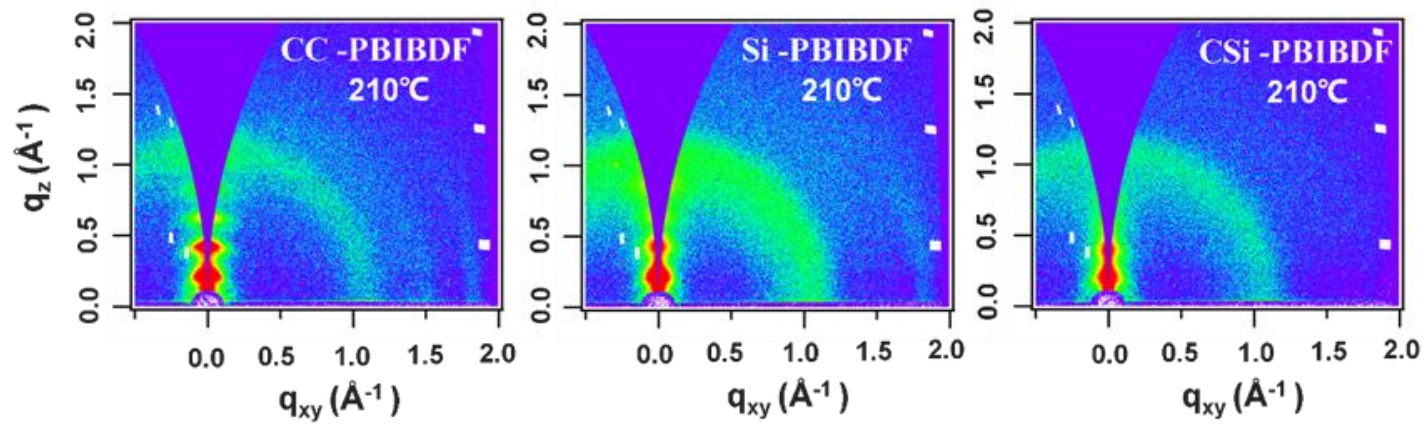

Figure S8. GIWAXS-2D patterns for as-cast polymer thin-films of CC-PBIBDF, SiPBIBDF, and CSi-PBIBDF. 

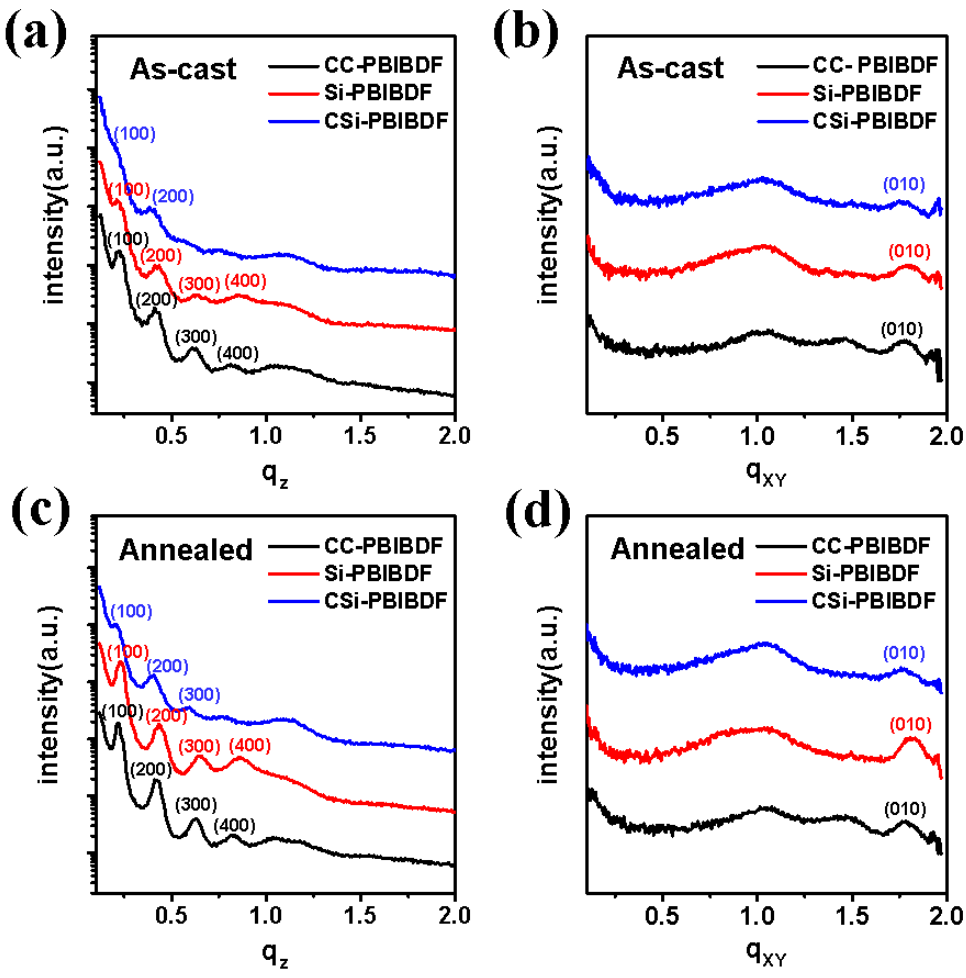

Figure S9. GIWAXS-1D curves of as-cast and annealed polymer thin-films of CCPBIBDF, Si-PBIBDF, and CSi-PBIBDF.
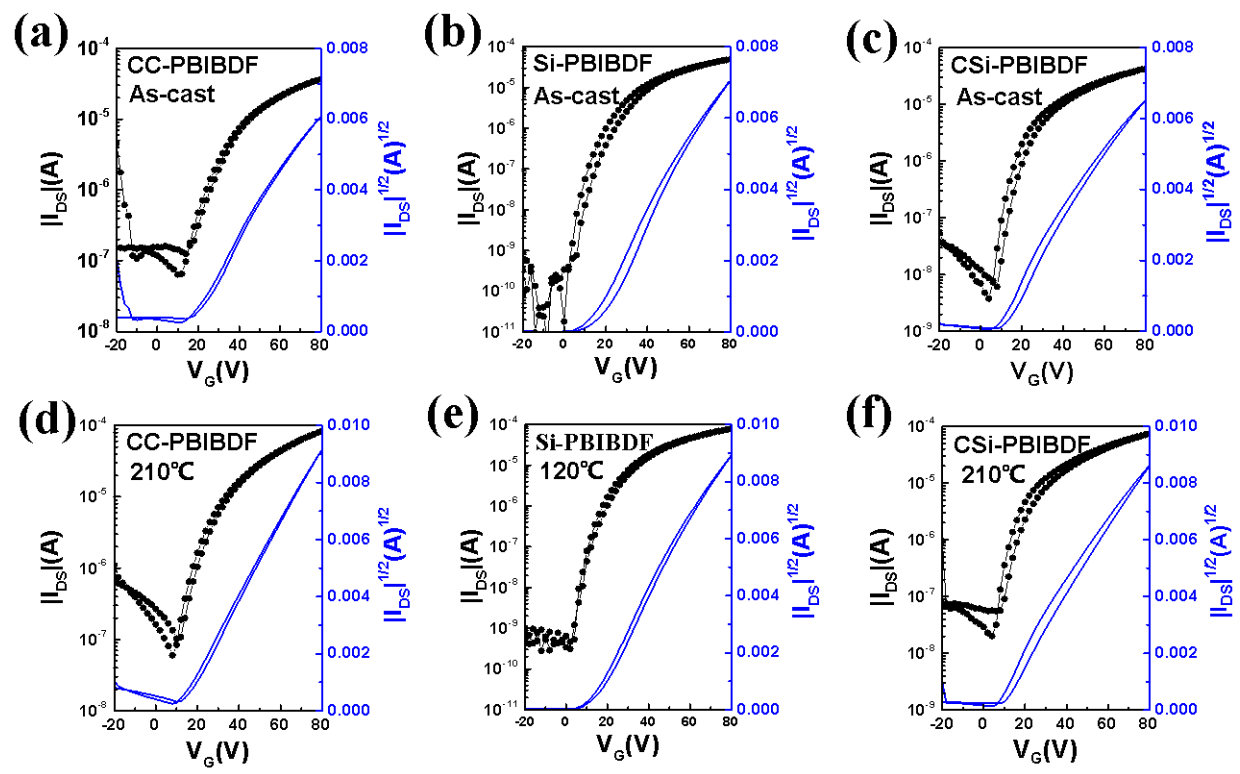

Figure S10.Transfer characteristic curves of OFETs based on the polymer thin-films of CC-PBIBDF, Si-PBIBDF, and CSi-PBIBDF. 

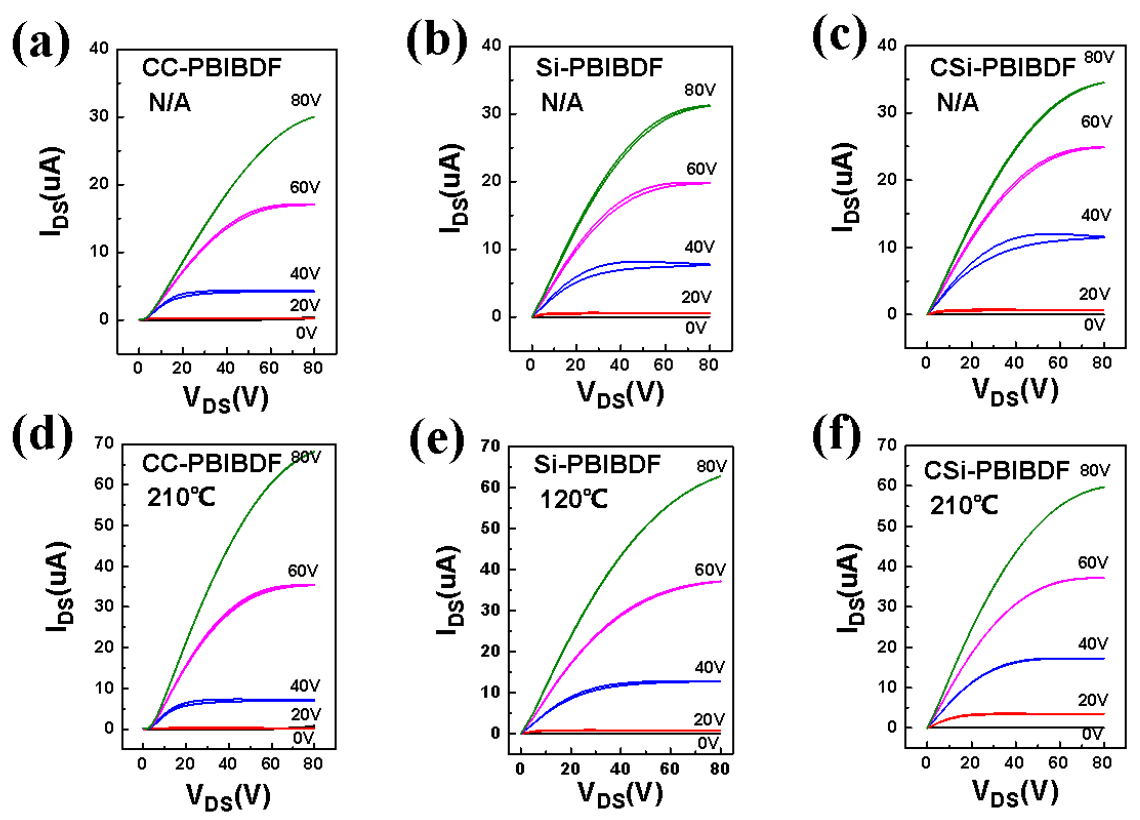

Figure S11. Output characteristic curves of OFETs based on the polymer thin-films of CC-PBIBDF, Si-PBIBDF, and CSi-PBIBDF.

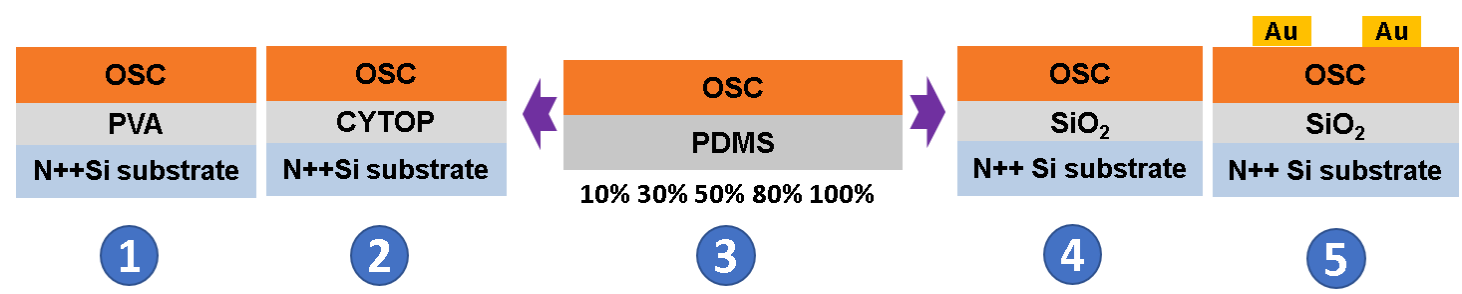

Figure S12. Fabrication process of OFETs with the stretched polymer films.
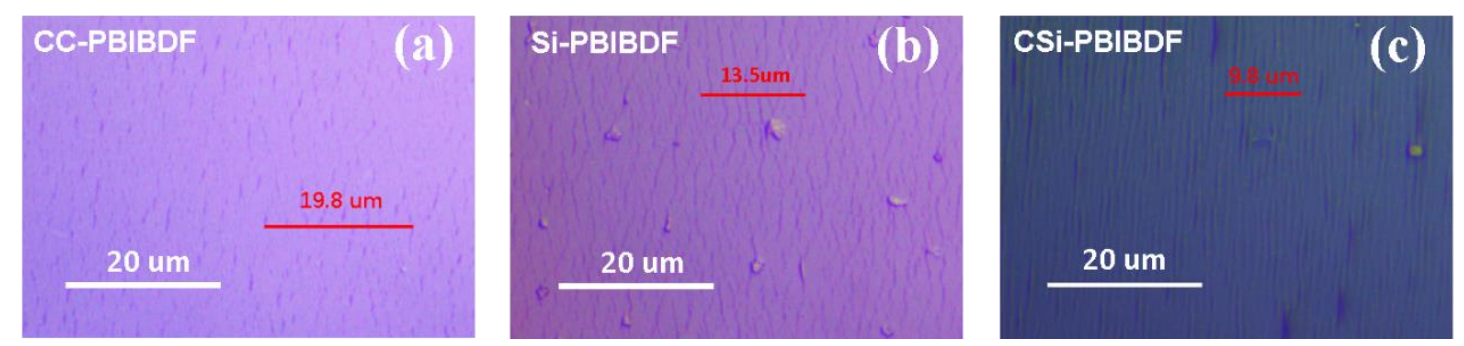

Figure S13. OM images of the polymer thin-films of (a) CC-PBIBDF, (b) Si-PBIBDF, and (c) CSi-PBIBDF on PDMS substrate. Polymer films approximately 40-50 nm thick were transferred onto the PDMS substrate. Once they were released from the prestrained PDMS, buckles were clearly observed. The modulus of the polymer semiconductors were determined based on the wavelength of the buckles, the thickness of the polymer films, and the modulus of the PDMS substrate. 

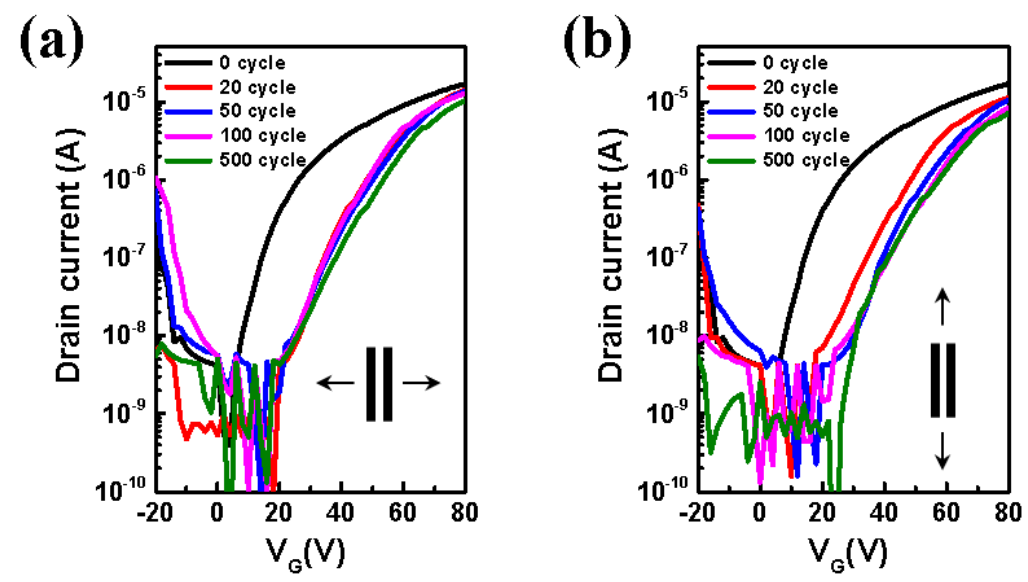

Figure S14. Transfer characteristic curves of the printed polymer thin-films of CSiPBIBDF after multiple stretching-releasing cycles (up to 500 cycles) at $50 \%$ strain parallel (a) and perpendicular (b) to strain direction.

Table S1. The calculation of the volume of different side chains in one repeat unit.

\begin{tabular}{|c|c|c|c|c|}
\hline $\begin{array}{c}\text { Type of } \\
\text { side chain }\end{array}$ & $\begin{array}{c}\text { cross-sectional } \\
\text { areas }\end{array}$ & $\begin{array}{c}\text { bond } \\
\text { length }\end{array}$ & $\begin{array}{c}\text { bond } \\
\text { angle }\end{array}$ & Volume of side chain in one repeat unit \\
\hline $\mathrm{V}_{\mathrm{LC}}$ & $21.5 \AA^{2}$ & $1.53 \AA$ & $110^{\circ}$ & $2 *\left(12 * 1.53 \AA * \sin 55^{\circ} * 21.5 \AA^{2}\right) \approx 645 \AA^{3}$ \\
\hline $\mathrm{V}_{\mathrm{BC}}$ & $43 \AA^{2}$ & $1.53 \AA$ & $110^{\circ}$ & $\begin{array}{r}2 *\left(10 * 1.53 \AA * \sin 55^{\circ} * 43 \AA^{2}\right)+2 *(2 * 1.53 \AA \\
\left.* \sin 55^{\circ} * 21.5 \AA^{2}\right) \approx 1182.5 \AA^{3}\end{array}$ \\
\hline $\mathrm{V}_{\mathrm{LSi}}$ & $41 \AA^{2}$ & $1.63 \AA$ & $150^{\circ}$ & $2 *\left(12 * 1.63 \AA * \sin 75^{\circ} * 41 \AA^{2}\right)+2 *(6 * 1.53 \AA$ \\
\end{tabular}




\section{Reference:}

S1. Ding, Y.; Jiang, L.; Du, Y.; Kim, S.; Wang, X.; Lu, H.; Zhang, G.; Cho, K.; Qiu, L. Linear hybrid siloxane-based side chains for highly soluble isoindigo-based conjugated polymers. Chem. Commun. 2020, 56, 11867-11870.

S2. Ding, Y.; Zhao, F.; Kim, S.; Wang, X.; Lu, H.; Zhang, G.; Cho, K.; Qiu, L. Azaisoindigo-Based Polymers with a Linear Hybrid Siloxane-Based Side Chain for High-Performance Semiconductors Processable with Nonchlorinated Solvents. ACS Appl. Mater. Interfaces 2020, 12, 41832-41841.

S3. Lei, T.; Dou, J. H.; Cao, X. Y.; Wang, J. Y.; Pei, J., A BDOPV-based donoracceptor polymer for high-performance n-type and oxygen-doped ambipolar fieldeffect transistors. Adv. Mater. 2013, 25, 6589-6593

S4. Zhang, G.; Li, P.; Tang, L.; Ma, J.; Wang, X.; Lu, H.; Kang, B.; Cho, K.; Qiu, L., A bis(2-oxoindolin-3-ylidene)-benzodifuran-dione containing copolymer for highmobility ambipolar transistors. Chem. Commun. 2014, 50, 3180-3183. 


\section{${ }^{1} \mathrm{H}$-NMR and ${ }^{13} \mathrm{C}$-NMR of monomers}

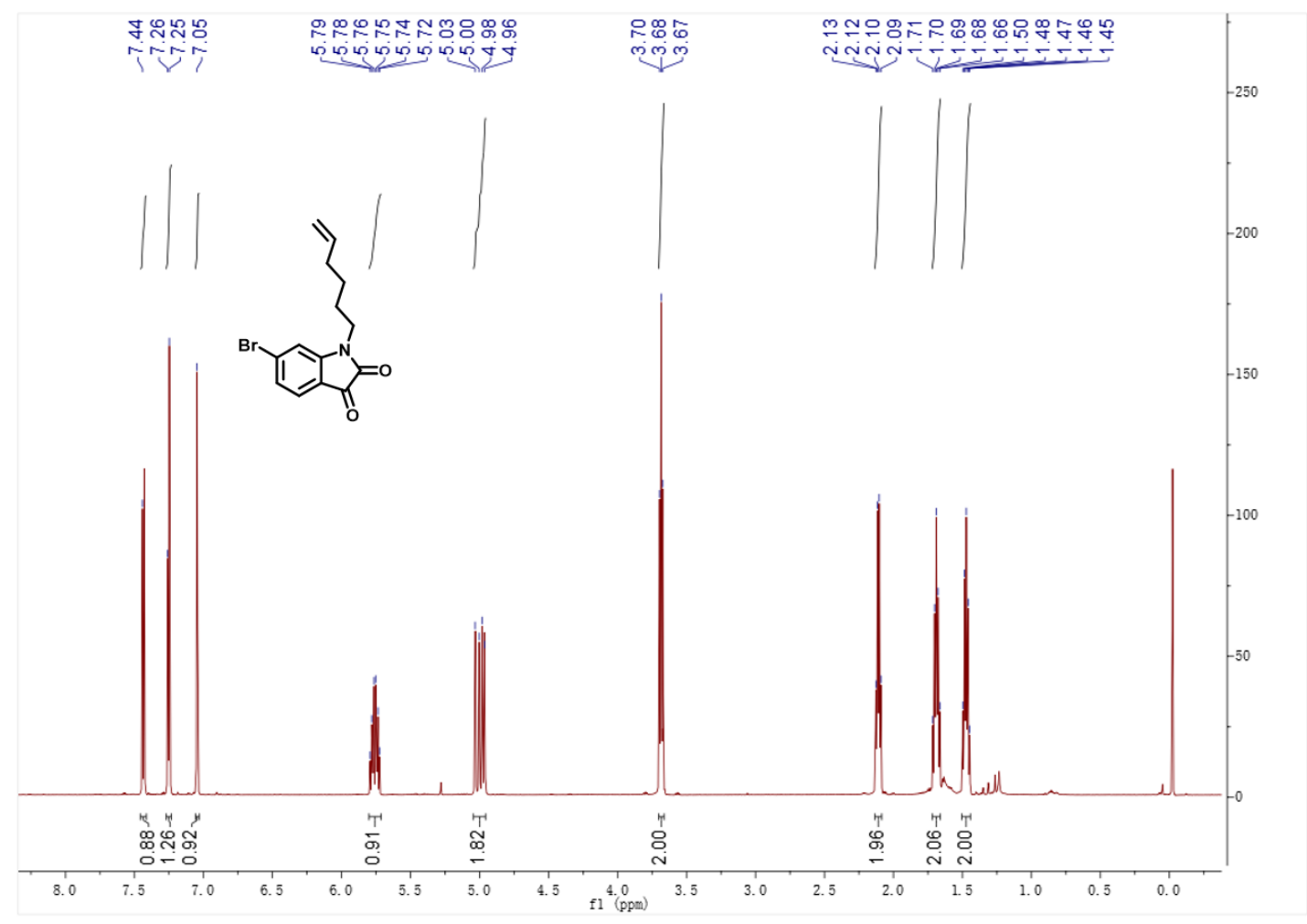

${ }^{1} \mathrm{H}$ NMR spectra of IDT-C.

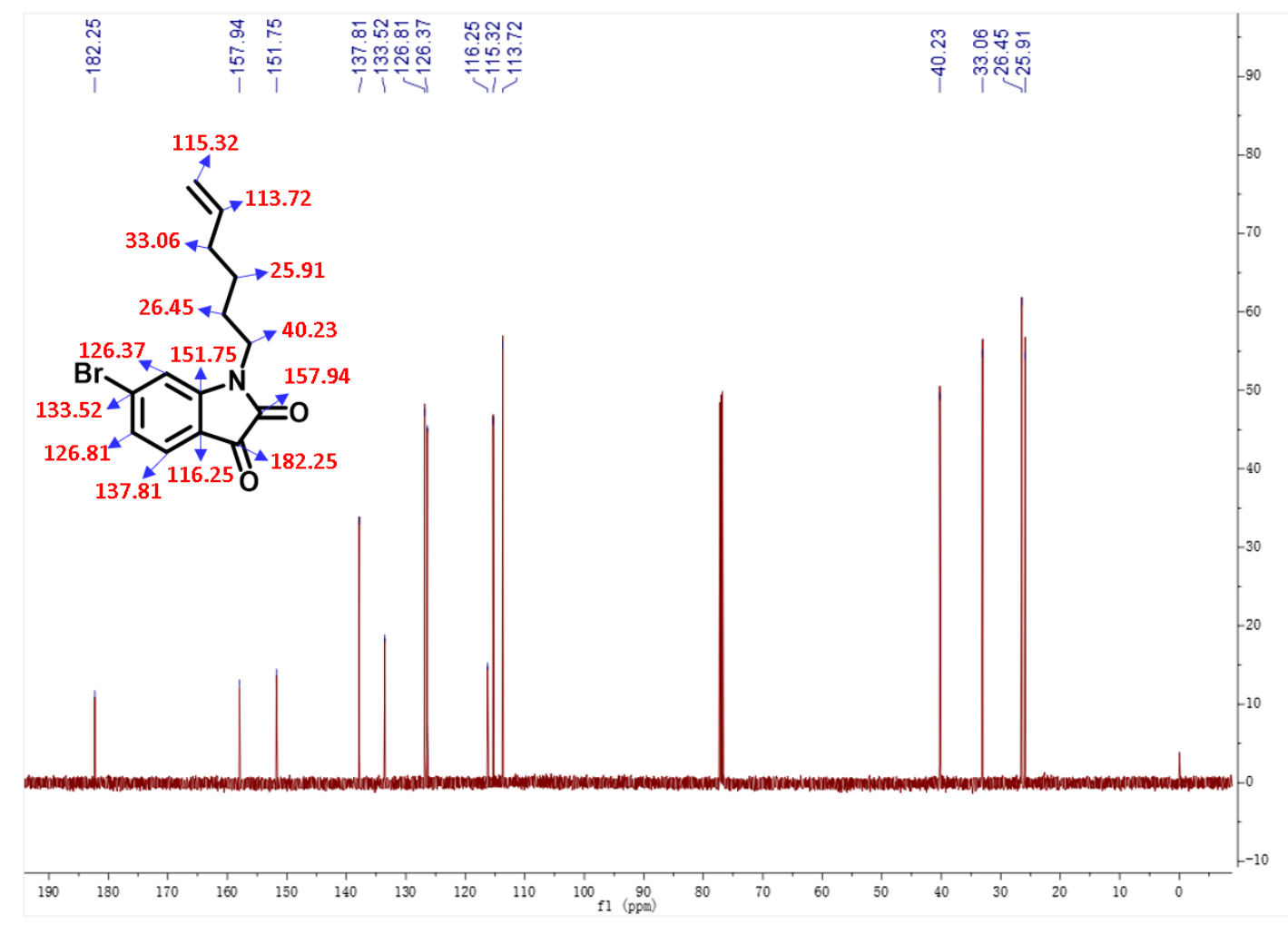

${ }^{13} \mathrm{C}$ NMR spectra of IDT-C. 


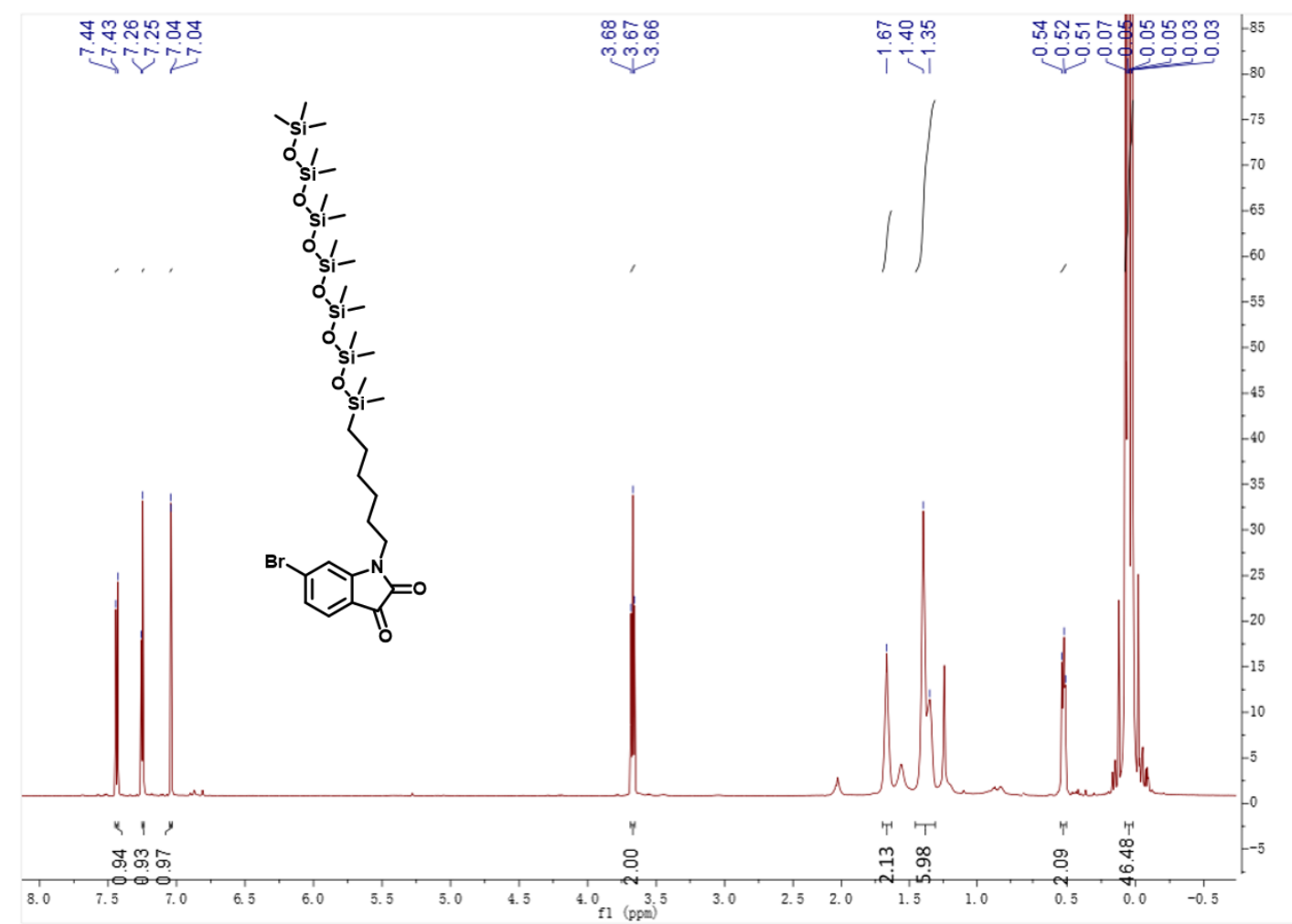

${ }^{1} \mathrm{H}$ NMR spectra of IDT-Si.

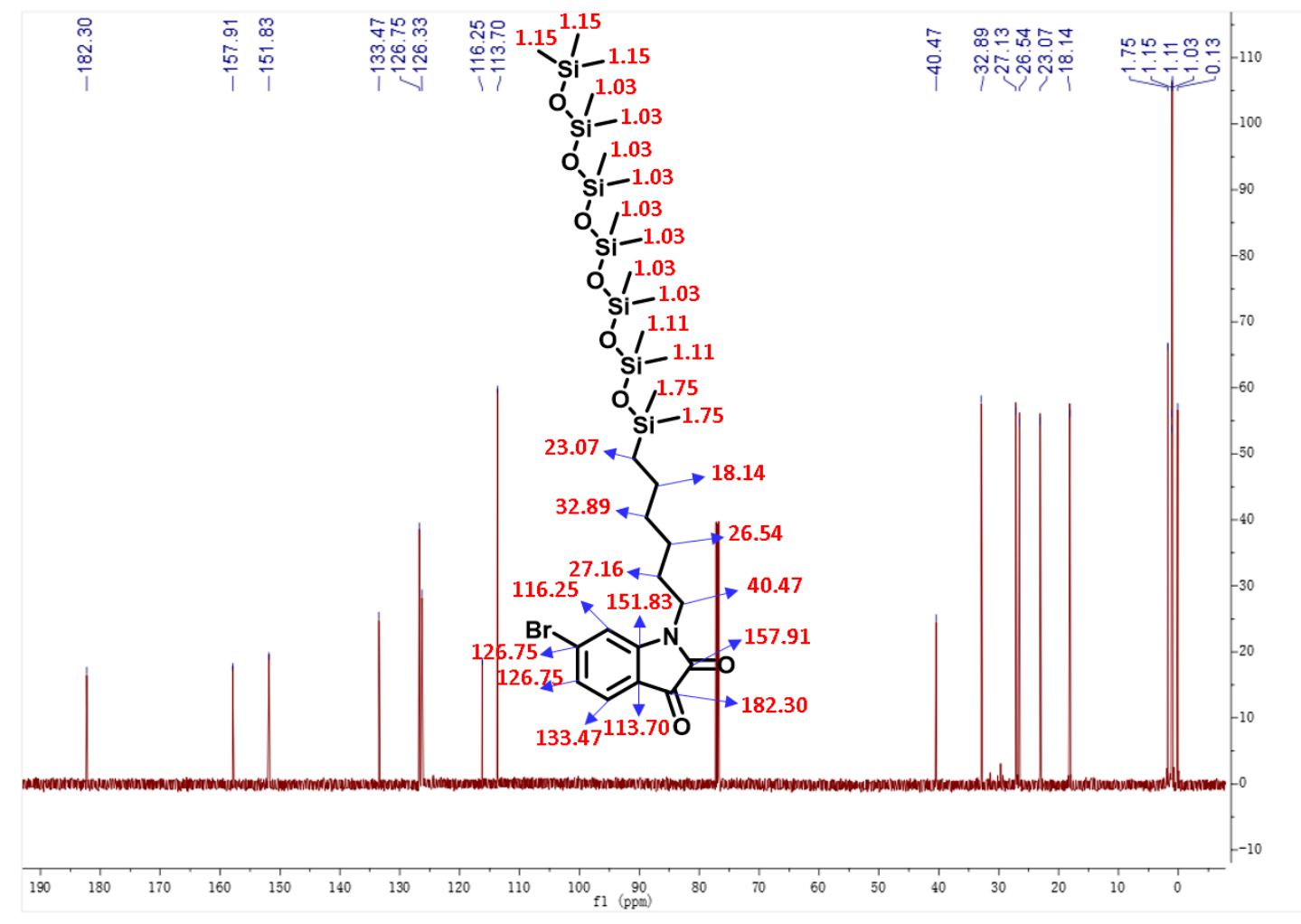

${ }^{13} \mathrm{C}$ NMR spectra of IDT-Si. 


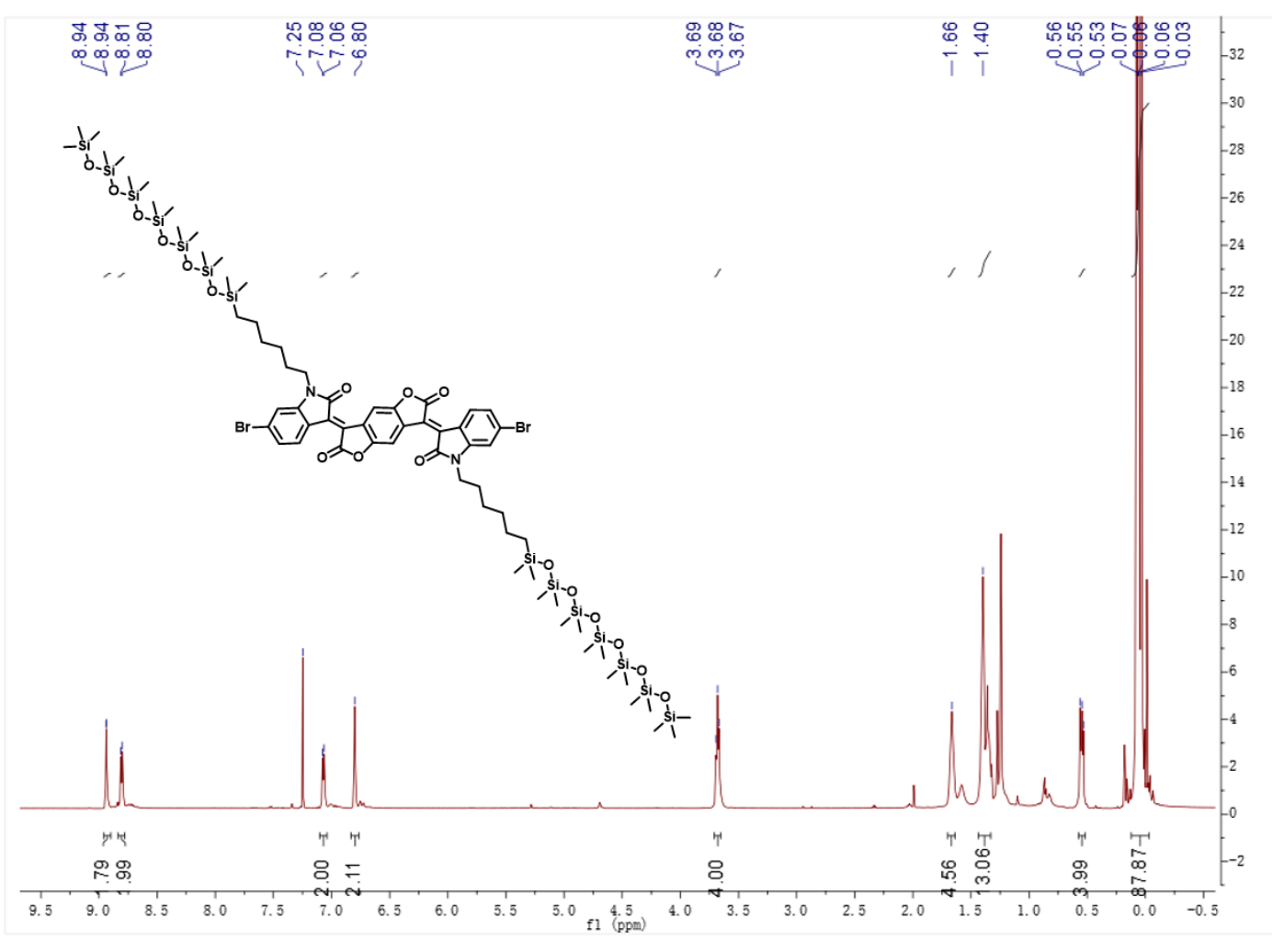

${ }^{1} \mathrm{H}$ NMR spectra of BIBDF-Si.

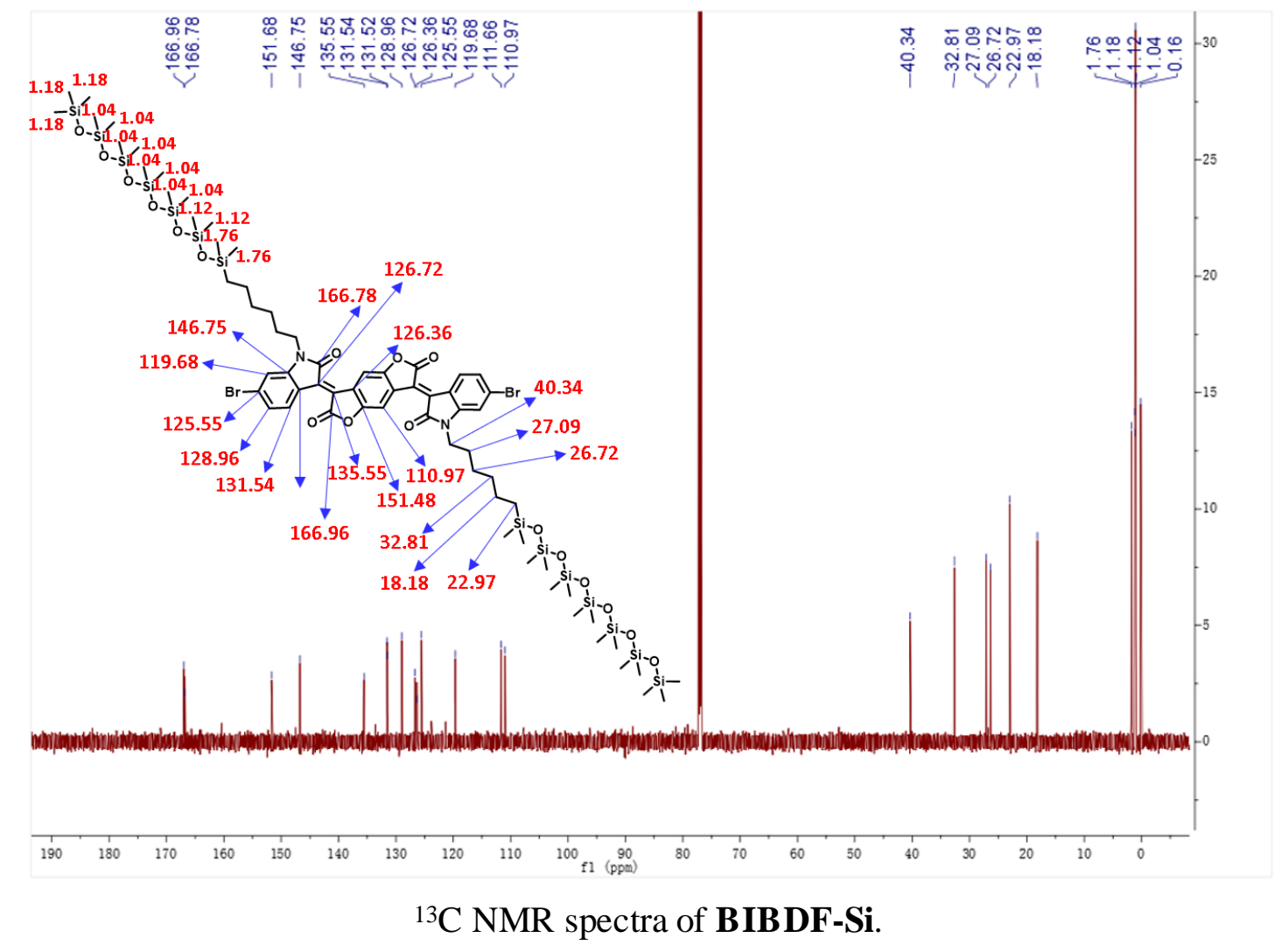


${ }^{29} \mathrm{Si}-\mathrm{NMR}$ of monomers

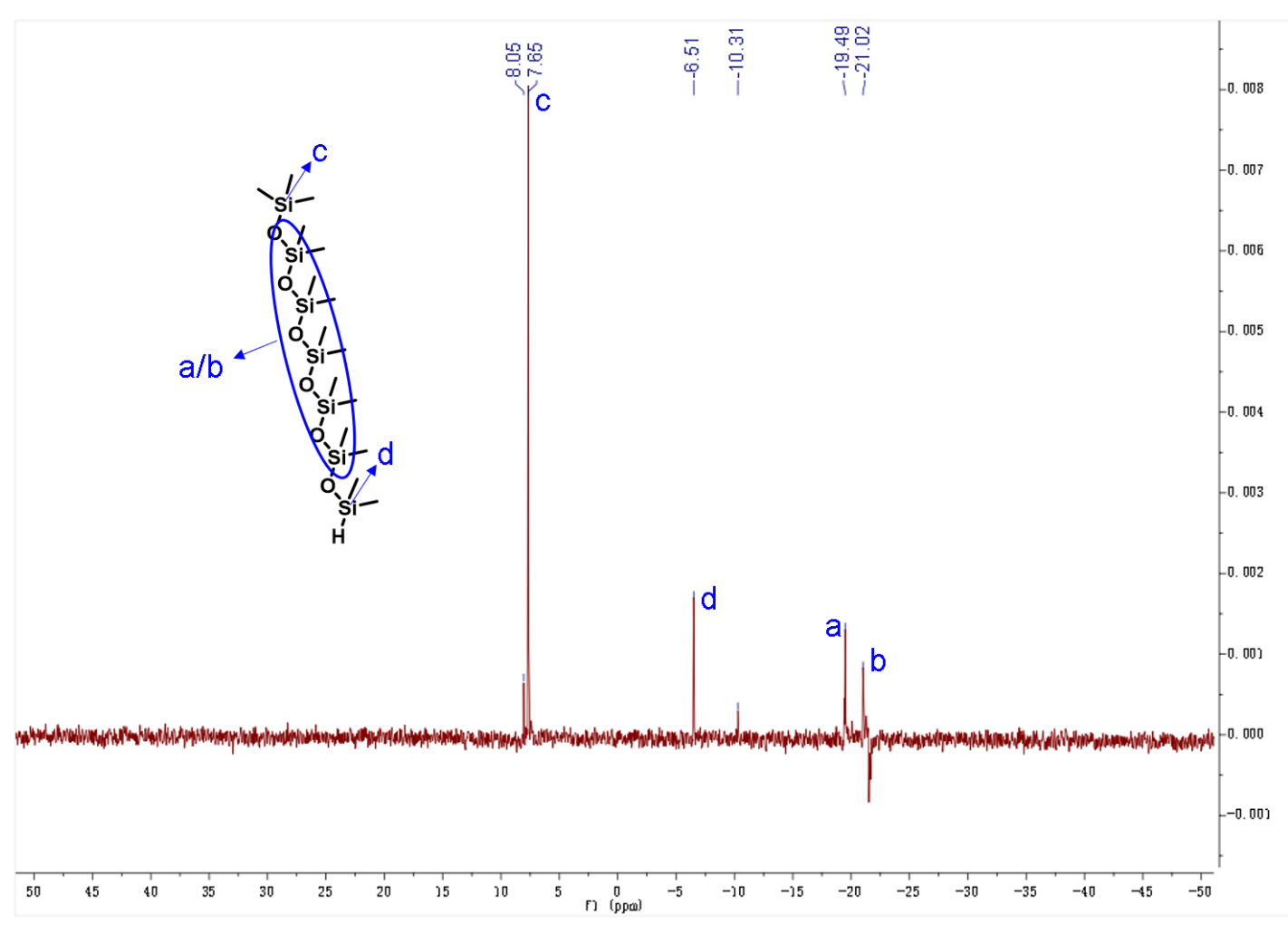

${ }^{29} \mathrm{Si}$ NMR spectra of pentadecamethylheptasiloxane

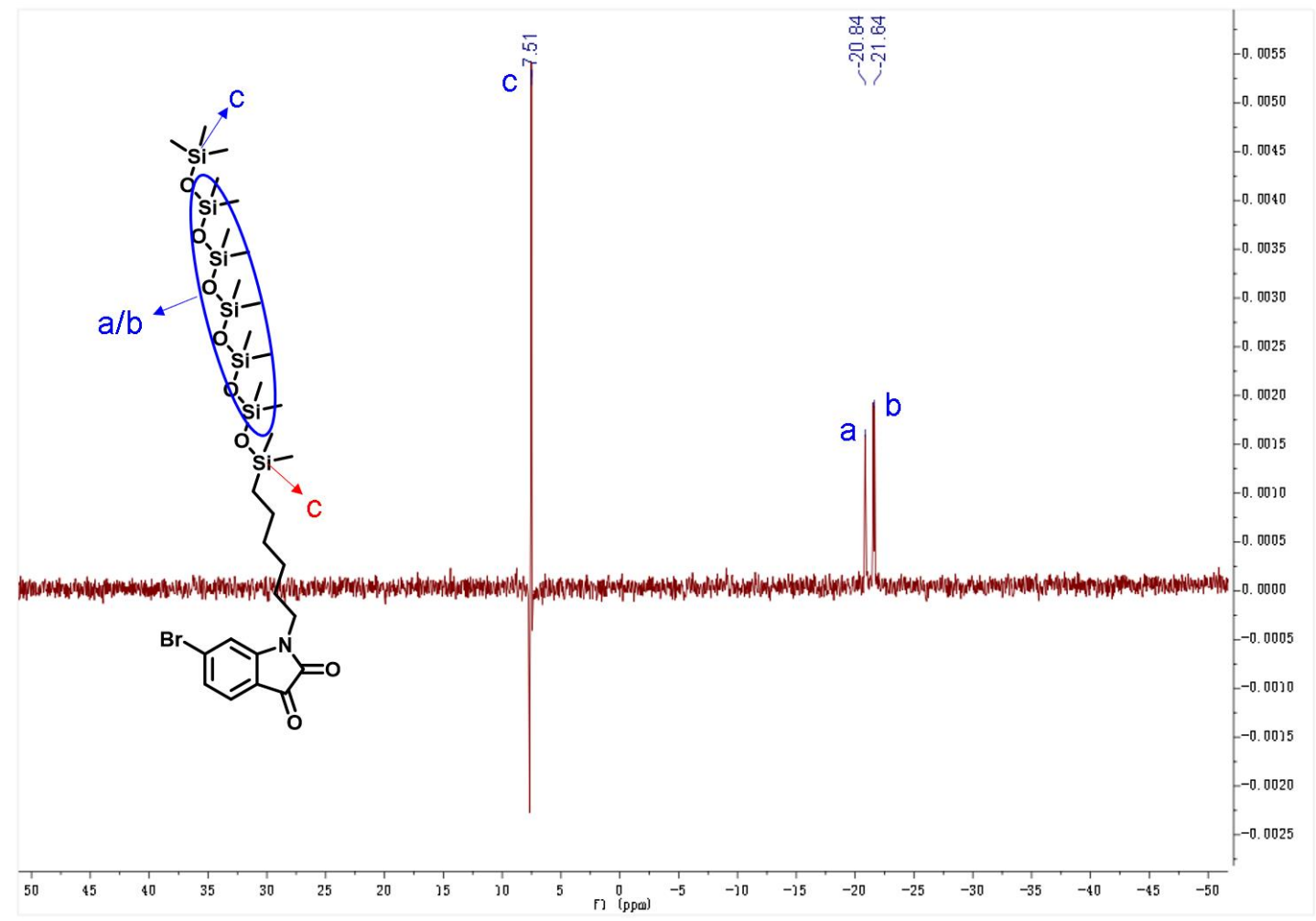

${ }^{29} \mathrm{Si}$ NMR spectra of IDT-Si 


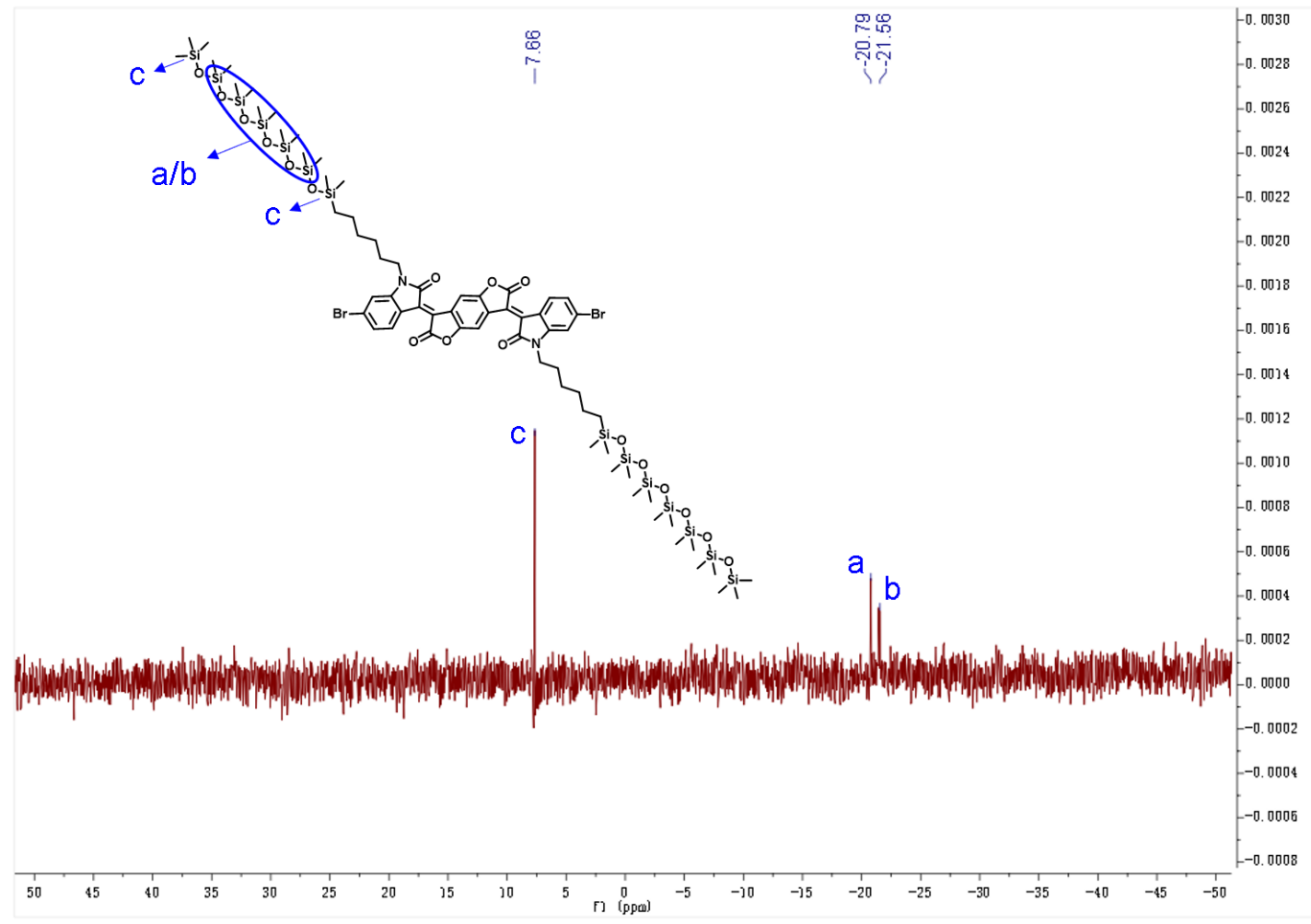

${ }^{29} \mathrm{Si}$ NMR spectra of BIBDF-Si

\section{${ }^{1} \mathrm{H}$-NMR of polymers}

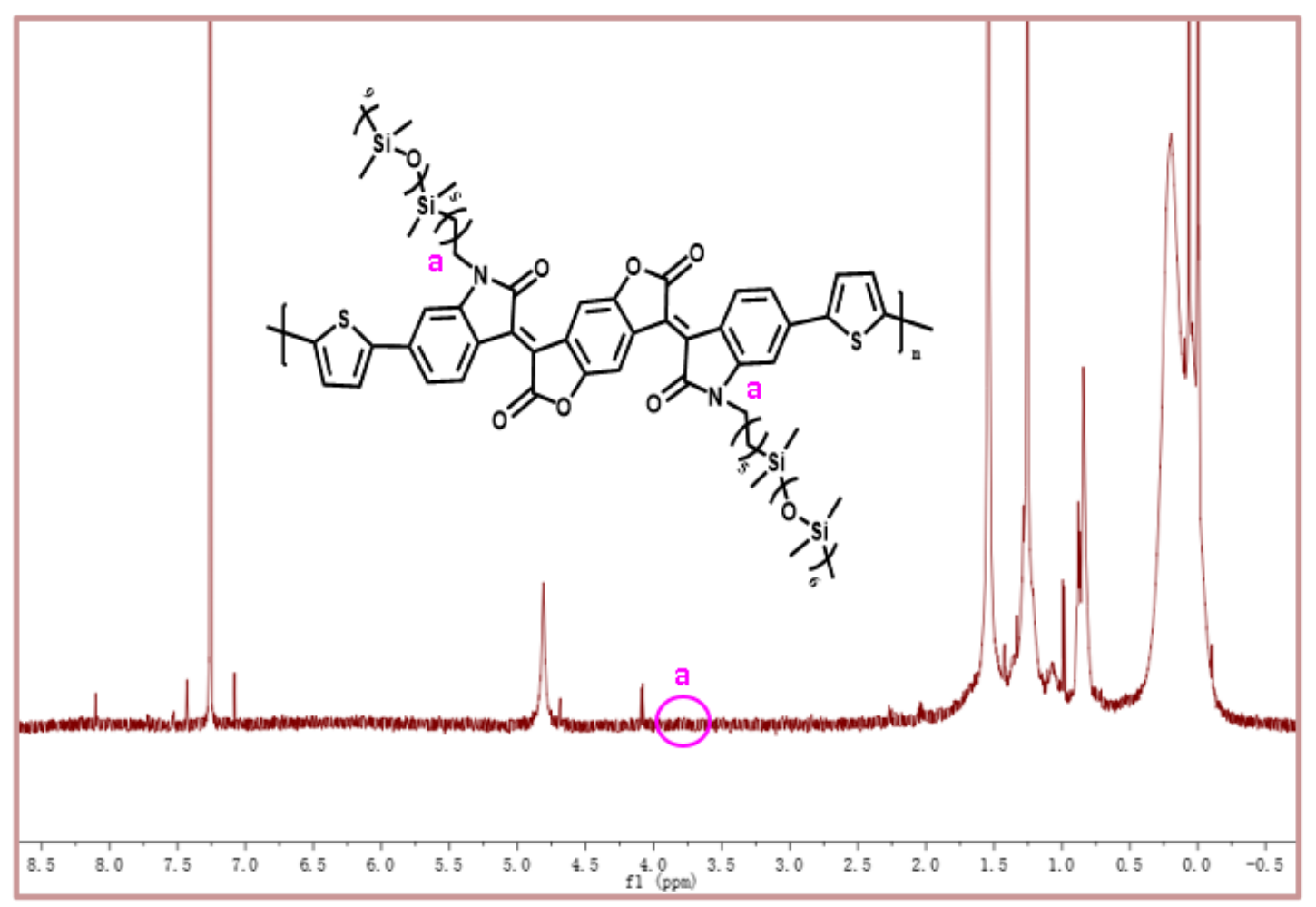

${ }^{1} \mathrm{H}$ NMR spectra of Si-PBIBDF. 


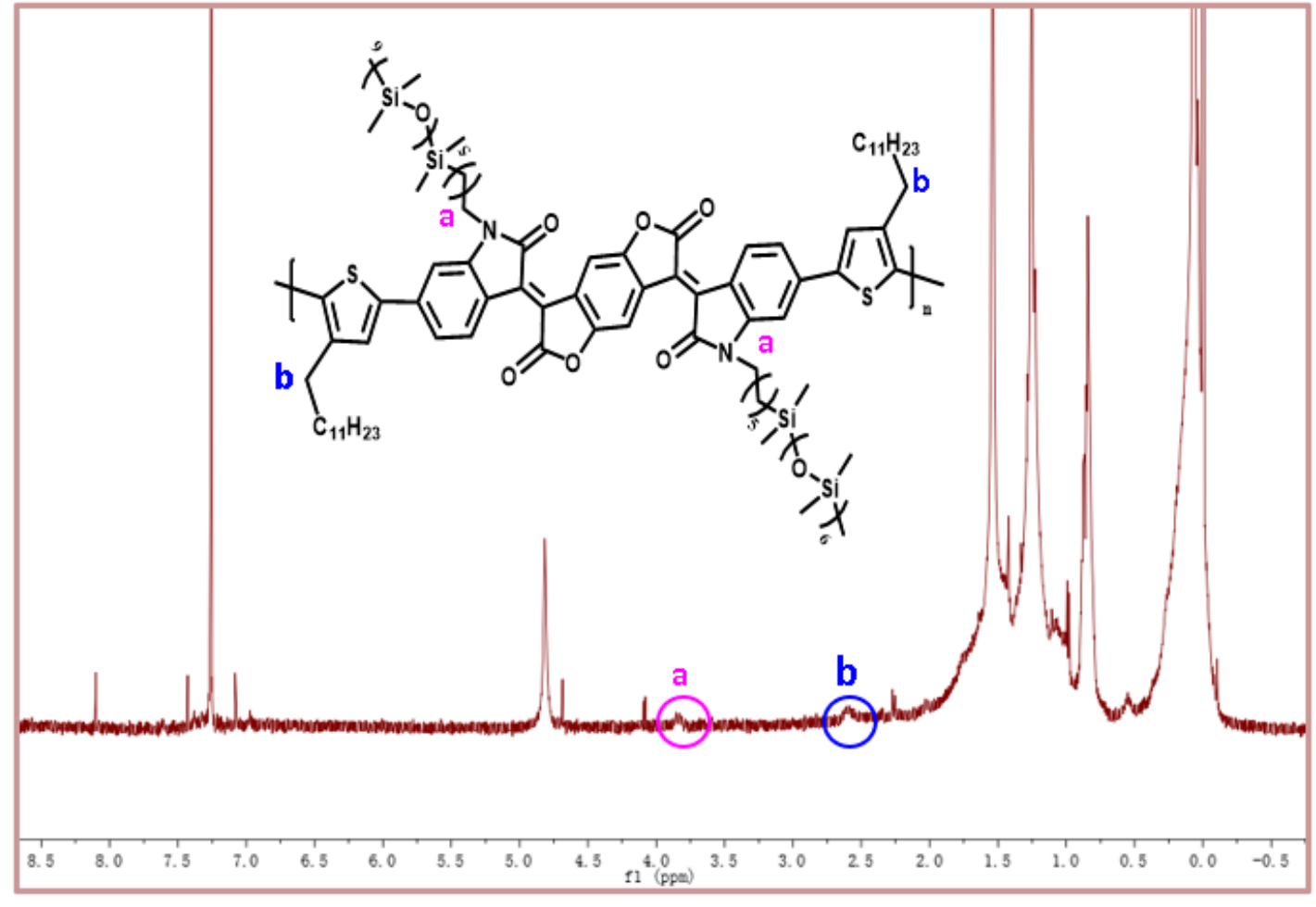

${ }^{1} \mathrm{H}$ NMR spectra of CSi-PBIBDF. 